\title{
Evaluation of the feasibility of using weekly paclitaxel as neoadjuvant therapy in patients with epithelial ovarian cancer; a pre-post clinical trial
}

\author{
Sakineh Ebrahimi ${ }^{\circledR}$, Seyed Saeed Hashemi Nazari ${ }^{\circledR}{ }^{\circledR}$, Arash Dooghaie Moghadam $^{2}{ }^{\circledR}$, Shirin $\operatorname{Haghighi}^{3^{*}(\mathbb{D}}$ \\ 'Loghman Hospital, Shahid Beheshti University of Medical Sciences, Tehran, Iran \\ ${ }^{2}$ Shahid Beheshti University of Medical Sciences, Tehran, Iran \\ ${ }^{3}$ Department of Oncology, Taleghani Hospital, Shahid Beheshti University of Medical Sciences, Tehran, Iran
}

\section{*Correspondence to}

Shirin Haghighi,

Email:drshirinhaghighi@sbmu ac.ir, shaghighi@taleghani-

hospital.ir

Received 2 April 2020 Accepted 10 June 2020 Published online 27 July 2020

Keywords: Ovarian neoplasm Neoadjuvant chemotherapy,

Antineoplastic agents, Paclitaxel, Carboplatin

\section{Abstract}

Introduction: Although weekly paclitaxel and carboplatin regimen is as effective as the standard method for treatment of advanced ovarian cancer, it has less frequently been used as neoadjuvant therapy.

Objectives: To reduce the side effects of typical every three-week chemotherapy and increase progression-free survival (PFS) rate, this study aimed to evaluate the feasibility of using weekly paclitaxel as neoadjuvant therapy in patients with epithelial ovarian cancer.

Patients and Methods: This pre-post clinical trial was conducted on 14 patients with stage IIIC (8 patients) and IV (6 patients) advanced ovarian carcinoma. All the patients received the three courses of treatment and then underwent interval debulking surgery. After the surgery, patients received three or five courses based on their stages. Every neoadjuvant chemotherapy course consisted of weekly paclitaxel $\left(80 \mathrm{mg} / \mathrm{m}^{2}\right)$ and carboplatin $(A \cup C=6)$ every 3 weeks. After every 21 days of treatment course, the patients were evaluated to investigate their response to treatment and side effects. Patients were followed up for at least 6 months.

Results: The mean (SD) age of the patients was $64 \pm 8$ years. After three courses of neoadjuvant chemotherapy, one patient $(7 \%)$ had a complete response and 13 patients $(93 \%)$ had a partial response. During the treatment period, two patients $(14 \%)$ developed anemia, one patient $(7 \%)$ developed neutropenia, two patients $(14 \%)$ developed thrombocytopenia, and six patients (43\%) developed neuropathy. The median (interquartile range) of PFS was 13 months (9.5-16.25)

Conclusion: The findings showed that a weekly paclitaxel and carboplatin regimen as neoadjuvant therapy could be effective in the treatment of advanced ovarian cancer. However, it is necessary to conduct multicenter studies with larger sample sizes.

Trial registration: Registration of trial protocol has been approved in Iranian Registry of Clinical Trial (identifier IRCT2017050333789N1; http://en.irct.ir/trial/25978)

Citation: Ebrahimi S,
Hashemi Nazari SS,
Dooghaie Moghadam A,
Haghighi S. Evaluation
of the feasibility of using
weekly paclitaxel as
neoadjuvant therapy in
patients with epithelial
ovarian cancer; a
pre-post clinical
trial. Immunopathol
Persa. 2021;7(1):e09.
DOI:10.34172/
ipp.2021.09.

\section{Introduction}

Ovarian cancer is the third most common malignancy in women worldwide, however, it is the deadliest cancer in women which kills 160000 people yearly (1). Its estimated annual incidence is 204000 cases all over the world (2). Since this cancer has few symptoms, the diagnosis is delayed until the final stages; as a result, $70 \%$ of the patients at the time of diagnosis are in advanced stages of the disease and this condition is associated with high morbidity and mortality (3). Thus, primary cytoreductive surgery is the standard treatment for the advanced types of this cancer $(4,5)$ and then chemotherapy is used. Currently, the recommended standard chemotherapy for ovarian cancer is a typical every three-week paclitaxel and carboplatin
Key point

Our study showed that a weekly paclitaxel and carboplatin regimen as neoadjuvant therapy could be as effective as its every three-week administration in the treatment of advanced ovarian cancer.

regimen for four or six consecutive courses. Neurotoxicity, thrombocytopenia, fever, and neutropenia are the common side effects of this regimen $(6,7)$. However, some studies have shown that weekly administration of paclitaxel at a low dose is as effective as every three-week administration of the drug $(8,9)$.

Neoadjuvant chemotherapy is the administration of chemotherapy prior to surgery which aims to reduce morbidity (complications) caused by the surgery.

\footnotetext{
Copyright (C) 2021 The Author(s); Published by Nickan Research Institute. This is an open-access article distributed under the terms of the Creative Commons Attribution License (http://creativecommons.org/licenses/by/4.0), which permits unrestricted use, distribution, and reproduction in any medium, provided the original work is properly cited.
} 
This type of treatment increases the chance of complete resection at the time of cytoreductive surgery and also improves the patient's surgical outcome (7). Typically, neoadjuvant therapy is recommended for patients who are not candidates for surgery because of the site of tumor, its size and extension, disease stage, or co-morbidities (7). According to a meta-analysis in 2006, neoadjuvant chemotherapy is associated with worse outcomes as compared with primary debulking surgery (10). However, recent studies have shown that this method is not inferior to primary debulking surgery and can be considered as an alternative treatment for patients with stage III or IV (11-16). Despite the effectiveness of neoadjuvant chemotherapy, the majority of aforementioned studies have investigated typical every three-week paclitaxel and carboplatin chemotherapy while few studies have assessed weekly paclitaxel therapy as neoadjuvant chemotherapy (17-20).

\section{Objectives}

To reduce the side effects of typical regimen (every 3-week chemotherapy) and increase progression-free survival (PFS) rate, this study aimed to evaluate the feasibility of using weekly paclitaxel as neoadjuvant therapy in patients with epithelial ovarian cancer.

\section{Patients and Methods}

Study design and participants

This clinical trial was conducted on all patients with advanced ovarian cancer who referred to Taleghani hospital in Tehran, Iran. The patients were in stage IIIc or higher and based on surgeon's diagnosis were not eligible for the surgery because of porta hepatis or mesentery root involvement, extended metastasis of the liver, and disseminated involvement of intestine. Based on the inclusion criteria, we recruited the patients who were over 18 years of age, had ovarian cancer confirmed by biopsy and pathology tests, were at the advanced stages of the disease confirmed by CT scan (stage IIIc, IV), did not receive any prior chemotherapy, had proper liver and kidney function, and were not candidate for primary surgery due to underlying disease. Overall, a total of 14 patients were enrolled in the study (Figure 1).

\section{Ethical issues}

The research followed the tenets of the Declaration of Helsinki. This paper was extracted from the thesis of Sakineh Ebrahimi, Department of internal medicine, Shahid Beheshti University of Medical Sciences. Moreover, the study protocol was registered in the Iranian Registry of Clinical Trials (identifier: IRCT2017050333789N1; http://en.irct.ir/trial/25978). Additionally, the study was approved by the ethics committee of the Shahid Beheshti University of Medical Sciences (\#IR.SBMU. MSP.Rec.1395.70). Accordingly, the process of the study was explained to the patients and informed consent was obtained from all of them.

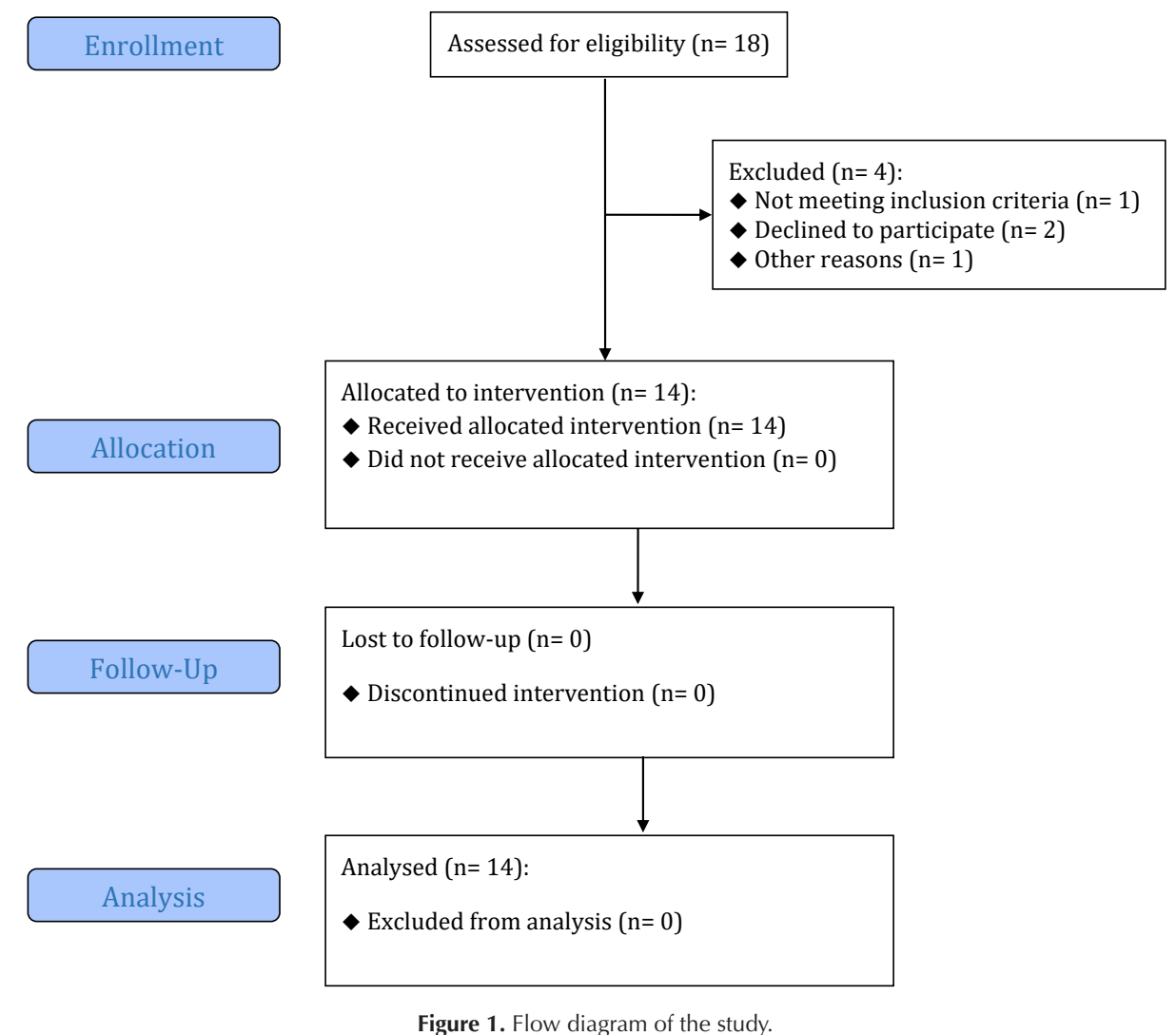




\section{Study protocol}

First, a checklist was used to record patients' data including age, characteristics of cancer (site of tumor, biopsy type, tumor pathology, histological grade of the tumor and tumor stage), the result of CT scan of the chest, size of peritoneum metastases, presence of ascites, and patient functioning status (based on history, physical examination, and Eastern Cooperative Oncology Group [ECOG] criteria (21)). Then, the patients underwent neoadjuvant chemotherapy. Each course of neoadjuvant chemotherapy regimen included every three-week carboplatin (in day 1) with $\mathrm{AUC}=6$ and weekly paclitaxel (in days 1,8 , and 15) with a dose of $80 \mathrm{mg} / \mathrm{m}^{2}$. Carboplatin was solved in 250 $\mathrm{mL}$ dextrose serum $5 \%$ and was infused within two hours. Paclitaxel was solved within $500 \mathrm{~mL}$ normal saline and was infused within 2 hours. All the patients received the three consecutive courses of treatment. Then, the patient underwent CT scan of the abdomen, pelvis, and lungs and the results were recorded in the checklist. The response evaluation criteria in solid tumor (RECIST 1.1) (22) were used to evaluate the patients and those with partial or complete response underwent interval-debulking surgery. After the surgery, patients with stage IIIC cancer received three other courses of chemotherapy and the other patients received five other courses of chemotherapy through the abovementioned method. After every 21 days of treatment course, the patients were evaluated to investigate their response to treatment and side effects (including anemia, thrombocytopenia, neutropenia, fever and neuropathy). Patients were followed up for at least six months. An increase of more than $20 \%$ in diameter of the largest known lesions, emergence of a new lesion, and elevation of cancer antigen 125 (CA-125) serum level to more than $50 \%$ as compared with the previous measured level were considered as the signs of relapse. Finally, the PFS rate was measured from start of treatment until relapse.

\section{Statistical analysis}

SPSS 22 software was used for data analysis. Mean and standard deviation or median (interquartile range) were used to describe quantitative variables and frequency and percentage were used to describe qualitative variables.

\section{Results}

The range and mean (SD) age of the patients were 4576 years and $64 \pm 8$ years, respectively. Table 1 shows different characteristics of cancer. Laparoscopic biopsy was the most common method of biopsy. The most common pathological subtype was serous. Considering the histologic grade of the disease, most of cases were poorly differentiated. Moreover, eight patients (57\%) were in Stage IIIC and six patients (43\%) were in Stage IV. The frequency of metastasis with size of $0-2 \mathrm{~cm}$ was more than the other types (Table 1).

At baseline, two patients (14\%) were at zero functional level, seven patients (50\%) were in the first functional level,
Table 1. Characteristics of advanced ovarian cancer

\begin{tabular}{|c|c|c|}
\hline & & No. $(\%)$ \\
\hline \multirow{3}{*}{ Cancer type } & Epithelial ovarian & $8(57 \%)$ \\
\hline & Peritoneal & $5(36 \%)$ \\
\hline & Fallopian tube & $1(7 \%)$ \\
\hline \multirow{3}{*}{ Biopsy type } & Laparoscopic & $7(50 \%)$ \\
\hline & FNA & $5(36 \%)$ \\
\hline & Image guidance & $2(14 \%)$ \\
\hline \multirow{4}{*}{ Pathology } & Serous & $7(50 \%)$ \\
\hline & Endometrioid & $2(14 \%)$ \\
\hline & Clear cell & $1(7 \%)$ \\
\hline & Others or unknown & $4(29 \%)$ \\
\hline \multirow{4}{*}{ Histologic grade } & Well differentiated & $2(14 \%)$ \\
\hline & Moderately differentiated & $5(36 \%)$ \\
\hline & Poorly differentiated & $6(43 \%)$ \\
\hline & Unknown & $1(7 \%)$ \\
\hline \multirow{2}{*}{ Stage } & IIIC & $8(57 \%)$ \\
\hline & IV & $6(43 \%)$ \\
\hline \multirow{6}{*}{$\begin{array}{l}\text { Peritoneal } \\
\text { metastasis size at } \\
\text { beginning }\end{array}$} & No metastasis & $3(22 \%)$ \\
\hline & $0-2 \mathrm{~cm}$ & $5(36 \%)$ \\
\hline & $2-5 \mathrm{~cm}$ & $2(14 \%)$ \\
\hline & $5-10 \mathrm{~cm}$ & $1(7 \%)$ \\
\hline & $10-20 \mathrm{~cm}$ & $2(14 \%)$ \\
\hline & Unknown & $1(7 \%)$ \\
\hline
\end{tabular}

and five patients (36\%) were in the second functional level. The results of chest CT scan were indicative of no lesion in nine patients (64\%), pleural effusion in four patients (29\%), and lymph node involvement in one patient (7\%). In addition, 12 patients (86\%) had ascites.

After three courses of neoadjuvant chemotherapy, based on RECIST 1.1. criteria, one patient (7\%) had a complete response and 13 patients (93\%) had a partial response. Overall, 10 patients $(71 \%)$ received six courses and four patients (29\%) received eight courses of chemotherapy. During the treatment period, two patients (14\%) developed anemia, one patient $(7 \%)$ developed neutropenia, two patients (14\%) developed thrombocytopenia, and six patients (43\%) developed neuropathy. The median PFS (interquartile range) was 13 months (9.5-16.25) and its mean $(\mathrm{SD})$ was $13 \pm 5.7$ months.

\section{Discussion}

The results of this study showed that of 14 patients with advanced ovarian cancer who underwent neoadjuvant chemotherapy, 13 patients (93\%) had a partial response after three courses of primary chemotherapy. Overall, 10 patients received six courses and four patients received eight courses of chemotherapy. Considering the side effects of treatment, two patients (14\%) developed anemia, one patient $(7 \%)$ developed neutropenia, two patients $(14 \%)$ developed thrombocytopenia, and six patients (43\%) developed neuropathy. The mean PFS in all patients was $13 \pm 5.7$ months.

The typical every three-week paclitaxel and carboplatin regimen is currently used as the standard treatment for 
advanced ovarian cancer. This treatment is effective and has a high response rate and long PFS and prolonged overall patient survival $(23,24)$. However, some studies have shown that weekly administration of paclitaxel is as effective as its every three-week administration $(8,9)$. Although this type of treatment is more frequently used as an adjuvant treatment, some studies have also investigated the use and efficacy of weekly paclitaxel as neoadjuvant therapy. Tabata et al investigated the safety and toxicity of a low-dose regimen of weekly paclitaxel and carboplatin as neoadjuvant or an adjuvant therapy in patients with gynecologic cancers (ovarian or endometrial) with venous thrombosis and stated that a low-dose regimen of weekly paclitaxel and carboplatin is an appropriate treatment for such cancers (18). Safra et al investigated the safety and the outcome of this type of treatment as neoadjuvant treatment. Based on their results, the median of PFS was 25.74 months and the overall and complete response rates were $92.1 \%$ and $64.1 \%$, respectively. It was also reported that the use of weekly paclitaxel and carboplatin regimen as primary chemotherapy for epithelial ovarian cancer is feasible and can be tolerated by the patients, however, more studies are needed. Safra et al not only administered paclitaxel on a weekly basis, but also administered carboplatin on a weekly basis too and the chemotherapies were performed in courses of 28 days (17). They also conducted a retrospective study in 2014 and compared the efficiency of these two methods as an adjuvant or neoadjuvant therapy. They reported that the use of a weekly regimen improved PFS, however, its overall survival was similar to that of three-week regimen (25). Alcarraz et al assessed the effect of neoadjuvant therapy using a dose-dense regimen. They reported that the administration of a dose-dense regimen as neoadjuvant therapy was highly effective in patients with ovarian cancer whose tumor could not be removed via primary surgery (26). The efficacy of a dose-dense regimen as neoadjuvant therapy in 74 patients was investigated by Takahiro et al. Based on their findings, the median PFS was 17.7 months. They concluded that the administration of this regimen as neoadjuvant chemotherapy was effective and tolerable for the treatment of advanced ovarian and fallopian tube cancers and primary peritoneal carcinoma (27). Accordingly, Becker et al in a retrospective study investigated the use of a dose-dense regimen as neoadjuvant therapy and compared it with typical regimen. The study showed that 21 patients received a dose dense regimen and 40 patients received a typical regimen as neoadjuvant therapy. They concluded that although the dose-dense regimen toxicity was higher, it was associated with higher pathologic complete response and lack of residual disease (20). Dessai et al conducted a study to investigate the efficacy and safety of weekly neoadjuvant chemotherapy in 11 patients who were not able to tolerate the typical three-week regimen. Life-threatening side effects grade 3 and 4 were observed in two patients. After treatment, the response rate was $100 \%$. Finally, it was concluded that the use of weekly paclitaxel and carboplatin regimen was safe and effective in patients who could not tolerate three-week regimen (19). In line with other studies, our results showed that after three courses of primary chemotherapy, $100 \%$ of the patients had partial or complete response. Considering the side effects occurring during the period of treatment, neuropathy was the most common complication and PFS median was 13 months.

\section{Conclusion}

The findings of this study showed that weekly paclitaxel and carboplatin regimen as neoadjuvant therapy was effective in treating advanced ovarian cancer and had $100 \%$ treatment response (complete or partial) after the first three courses. In addition, the side effects were tolerable and the median of PFS was 13 months. However, in order to use this method as an alternative to the standard method, it is recommended to conduct further studies.

\section{Limitations of the study}

Our study was a preliminary study with a small sample size which lacked a control group; however, it showed that weekly paclitaxel and carboplatin regimen could be used as neoadjuvant therapy and had proper outcomes. Nevertheless, to use this method as an alternative to standard method, it is recommended to conduct multicenter studies with larger sample sizes and with control groups to verify the results.

\section{Authors' contribution}

$\mathrm{SE}$ and $\mathrm{SH}$ participated in the design and conduct of the study and also preparing the manuscript draft. SSH and ADM participated in describing the methodology of the study and statistical analysis of the data and amending the manuscript draft of the article. All authors read and approved the final manuscript.

\section{Conflicts of interest}

The authors declared no competing interests.

Ethical considerations

Ethical issues including plagiarism, double publication, and redundancy have been completely observed by the authors.

\section{Funding/Support}

This research was supported by Shahid Beheshti University of Medical Sciences. This study has been extracted from the thesis of Mrs. Sakineh Ebrahimi in school of medicine at this University (Grant \#128).

\section{References}

1. Lozano R, Naghavi M, Foreman K, Lim S, Shibuya K, Aboyans $\mathrm{V}$, et al. Global and regional mortality from 235 causes of death for 20 age groups in 1990 and 2010: a systematic analysis for the Global Burden of Disease Study 2010. The Lancet 2013;380:2095-128. doi: 10.1016/S0140-6736(12)61728-0

2. Sankaranarayanan R, Ferlay J. Worldwide burden of gynaecological cancer: the size of the problem. Best Pract Res Clin Obstet Gynaecol 2006;20:207-25. doi: 10.1016/j. bpobgyn.2005.10.007

3. Jemal A, Siegel R, Xu J, Ward E. Cancer statistics, 2010. CA Cancer J Clin 2010;60:277-300. doi: 10.3322/caac.20073 
4. Griffiths CT, Fuller AF. Intensive surgical and chemotherapeutic management of advanced ovarian cancer. Surg Clin North Am 1978;58:131-42. doi: 10.1016/S0039-6109(16)41440-4

5. Du Bois A, Quinn M, Thigpen T, Vermorken J, Avall-Lundqvist E, Bookman M, et al. 2004 consensus statements on the management of ovarian cancer: final document of the $3 \mathrm{rd}$ International Gynecologic Cancer Intergroup Ovarian Cancer Consensus Conference (GCIG OCCC 2004). Ann Oncol 2005;16:viii7-12. doi: 10.1093/annonc/mdi961

6. Pignata S, De Placido S, Biamonte R, Scambia G, Di Vagno G, Colucci G, et al. Residual neurotoxicity in ovarian cancer patients in clinical remission after first-line chemotherapy with carboplatin and paclitaxel: the Multicenter Italian Trial in Ovarian cancer (MITO-4) retrospective study. BMC Cancer 2006;6:5. doi: 10.1186/1471-2407-6-5

7. Vasey PA, Jayson GC, Gordon A, Gabra H, Coleman R, Atkinson $\mathrm{R}$, et al. Phase III randomized trial of docetaxel-carboplatin versus paclitaxel-carboplatin as first-line chemotherapy for ovarian carcinoma. J Natl Cancer Inst 2004;96:1682-91. doi: 10.1093/jnci/djh323

8. Markman M, Hall J, Spitz D, Weiner S, Carson L, Van Le $\mathrm{L}$, et al. Phase II trial of weekly single-agent paclitaxel in platinum/paclitaxel-refractory ovarian cancer. J Clin Oncol 2002;20:2365-69. doi: 10.1200/JCO.2002.09.130

9. Rose PG, Smrekar M, Fusco N. A phase II trial of weekly paclitaxel and every 3 weeks of carboplatin in potentially platinum-sensitive ovarian and peritoneal carcinoma. Gynecol Oncol 2005;96:296-300. doi: 10.1016/j.ygyno.2004.03.046

10. Bristow RE, Chi DS. Platinum-based neoadjuvant chemotherapy and interval surgical cytoreduction for advanced ovarian cancer: a meta-analysis. Gynecol Oncol 2006;103:1070-76. doi: 10.1016/j.ygyno.2006.06.025

11. Vergote I, Tropé CG, Amant F, Kristensen GB, Ehlen T, Johnson $\mathrm{N}$, et al. Neoadjuvant chemotherapy or primary surgery in stage IIIC or IV ovarian cancer. N Engl J Med 2010;363:94353. doi: 10.1056/NEJMoa0908806

12. Zheng $\mathrm{H}$, Gao Y-N. Primary debulking surgery or neoadjuvant chemotherapy followed by interval debulking surgery for patients with advanced ovarian cancer. Chin J Cancer Res 2012;24:304-09. doi: 10.1007/s11670-012-0268-7

13. Rauh-Hain JA, Rodriguez N, Growdon WB, Goodman A, Boruta DM, Horowitz NS, et al. Primary debulking surgery versus neoadjuvant chemotherapy in stage IV ovarian cancer. Ann Surg Oncol 2012;19:959-65. doi: 10.1245/s10434-0112100-x

14. Kehoe S, Hook J, Nankivell M, Jayson GC, Kitchener H, Lopes T, et al. Primary chemotherapy versus primary surgery for newly diagnosed advanced ovarian cancer (CHORUS): an openlabel, randomised, controlled, non-inferiority trial. The Lancet 2015;386:249-57. doi: 10.1016/S0140-6736(14)62223-6

15. Bian C, Yao K, Li L, Yi T, Zhao X. Primary debulking surgery vs. neoadjuvant chemotherapy followed by interval debulking surgery for patients with advanced ovarian cancer. Arch Gynecol Obstet 2016;293:163-68. doi: 10.1007/s00404-0153813-z
16. Sato S, Itamochi H. Neoadjuvant chemotherapy in advanced ovarian cancer: latest results and place in therapy. Ther Adv Med Oncol 2014;6:293-304. doi: 10.1177/1758834014544891

17. Safra T, Menczer J, Bernstein RM, Shpigel S, Matcejevsky D, Inbar MJ, et al. Combined weekly carboplatin and paclitaxel as primary treatment of advanced epithelial ovarian carcinoma. Gynecol Oncol 2009;114:215-18. doi: 10.1016/j. ygyno.2009.04.008

18. Tabata T, Tanida K, Umekawa T, Shiozaki T, Kondo E, Nagao $\mathrm{K}$, et al. Weekly low-dose paclitaxel and carboplatin therapy in gynecological cancer patients with venous thrombosis. Anticancer Res 2008;28:3971-75.

19. Dessai S, Chakraborty S, Babu T, Nayanar S, Bhattacharjee A, Jones J, et al. Tolerance of weekly metronomic paclitaxel and carboplatin as neoadjuvant chemotherapy in advanced ovarian cancer patients who are unlikely to tolerate 3 weekly paclitaxel and carboplatin. South Asian J Cancer 2016;5:63. doi: 10.4103/2278-330X.181629

20. Becker DA, Thomas ED, Gilbert AL, Boone JD, Straughn JM, Huh WK, et al. Improved outcomes with dose-dense paclitaxel-based neoadjuvant chemotherapy in advanced epithelial ovarian carcinoma. Gynecol Oncol 2016;142:25-9. doi: 10.1016/j.ygyno.2016.04.539

21. Oken MM, Creech RH, Tormey DC, Horton J, Davis TE, McFadden ET, et al. Toxicity and response criteria of the Eastern Cooperative Oncology Group. Am J Clin Oncol 1982;5:64956. doi: 10.1097/00000421-198212000-00014

22. Eisenhauer E, Therasse P, Bogaerts J, Schwartz L, Sargent $D$, Ford $R$, et al. New response evaluation criteria in solid tumours: revised RECIST guideline (version 1.1). Eur J Cancer 2009;45:228-47. doi: 10.1016/j.ejca.2008.10.026

23. Thigpen JT, Blessing JA, Ball H, Hummel SJ, Barrett RJ. Phase II trial of paclitaxel in patients with progressive ovarian carcinoma after platinum-based chemotherapy: a Gynecologic Oncology Group study. J Clin Oncol 1994;12:1748-53. doi: 10.1200/JCO.1994.12.9.1748

24. Seewaldt VL, Greer BE, Cain JM, Figge DC, Tamimi HK, Brown WS, et al. Paclitaxel (Taxol) treatment for refractory ovarian cancer: phase II clinical trial. Am J Obstet Gynecol 1994;170:1666-71. doi: 10.1016/S0002-9378(12)91832-3

25. Safra T, Shamai S, Greenberg J, Veizman A, Shpigel S, Matcejevsky D, et al. Weekly carboplatin with paclitaxel compared to standard three-weekly treatment in advanced epithelial ovarian carcinoma-a retrospective study. Gynecol Oncol 2014;132:18-22. doi: 10.1016/j.ygyno.2013.06.013

26. Alcarraz C, Olivera M, Muniz J, Morante Z, Ruiz R, Valdiviezo $\mathrm{N}$, et al. Carboplatin and paclitaxel dose dense as neoadjuvant chemotherapy follow by interval cytoreduction in advanced ovarian cancer. J Clin Oncol. 2015;33:e16553.

27. Takahiro E, Kodaira M, Shimoi T, Shimomura A, Yunokawa M, Yonemori K, et al. 2751 Dose-dense paclitaxel plus carboplatin as neoadjuvant chemotherapy for advanced ovarian, fallopian, and primary peritoneal carcinomas. Eur J Cancer 2015;51:S547. doi: 10.1016/S0959-8049(16)31517-9 